\title{
Maximum Oscillation Amplitude of Cargo Caused by Inertia Forces for Tower Cranes
}

\author{
Mingxiao Dong \\ Mechatronic Engineering School \\ Shandong Jianzhu University \\ Jinan, China \\ Bo Pang \\ Mechatronic Engineering School \\ Shandong Jianzhu University \\ Jinan, China
}

\begin{abstract}
In order to study the oscillation of the tower crane cargoes and to find the theories and methods for restraining the cargo oscillation and accurately positioning, in this paper we studied the effect of the dynamical parameters of the tower cranes on the center line of the cargo oscillation. The formulae to calculating the cargo oscillation caused by the inertia forces of mechanism motion are deduced to estimate the maximum oscillation amplitude of the cargoes.
\end{abstract}

Keywords-tower crane; cargo; inertia force;mechanism; oscillation

\section{INTRODUCTION}

Tower Cranes has three kinds of basic working conditions including transition, rotation and hoisting movement. In order to improve the working efficiency, it usually carries out the three motions at the same time. This makes cargo movement more complex. Namely, the cargoes do space pendulum with variable pendulum length and the suspension point moves along with the cargoes. The oscillation models are a two order nonlinear time varying differential equation [1-3]. In this paper, we studied the action of the inertia forces of the mechanism motions on the oscillation center line of the cargoes and deduced the formula calculating the maximum oscillation angle[4-6].

\section{NONLINEAR MODELS OF TOWER CRANES}

Tower cranes transport cargoes by the rotation of crane boom and trolley motion along the cargo boom. According to the motion characteristics, we set up a polar coordinate system $\{e \rho, e \psi\}$ whose coordinate origin is located at the intersection of the rotary center line of the tower body and the rotary surface of the crane cargo boom, non-inertial Cartesian coordinate system $\{\mathrm{i}, \mathrm{j}, \mathrm{k}\}$, and spherical coordinate system $\{\mathrm{e} \theta$, e $\varphi$, el $\}$ whose coordinate origin located at the suspension point of the hoisting cable and the suspension point moves with the trolley and synchronously rotates with the cargo boom. The structure schematic diagram and the coordinate systems of tower crane are shown in Figure 1 and Fig .2.

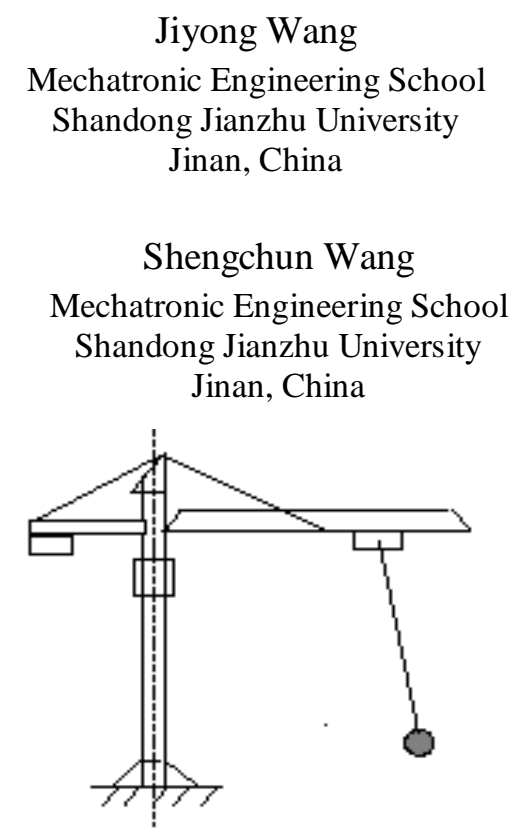

Figure 1. The structure schematic diagram of tower cranes.

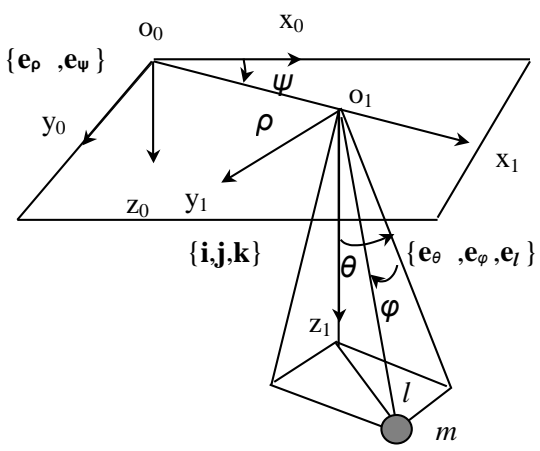

Figure 2. Tower crane coordinate systems.

The cargo hanging point in the polar coordinate system is the position of $(\rho, \psi), \rho$ and $\psi$ respectively denote the displacement of the trolley and the rotation angle of the cargo boom. The positions of the cargo in the non-inertial spherical coordinates are described with the three generalized coordinates $(1, \theta, \varphi)$, where 1 denotes the hoisting rope length, $\varphi$ denotes the angle between the hoisting rope and $\mathrm{x} 101 \mathrm{z} 1$ plane, $\theta$ is the angle between the projection of the hoisting cable in x1o1z1 plane and plumb line through the cargo hanging point. The crane system has five parameters which are the trolley velocity $\dot{\rho}$ or acceleration $\ddot{\rho}$, the rotary angular velocity $\dot{\psi}$ of the boom or the angular acceleration $\ddot{\psi}$, payload lifting velocity $\dot{l}$, or acceleration $\ddot{l}$, payload swing angles $\theta$ and $\varphi$. In this paper, 
the first three variables are control variables and the latter two are controlled variables.

According to the actual situation of crane work we gave the following assumptions and requirements: the mass of lifting cable can be negligible relative to the mass of hook and cargo, the stiffness of the lifting cable is sufficiently large and the length of the cable is negligible, the air resistance is negligible, sway angle of the cargoes is less than $10^{\circ}$.The nonlinear dynamic models of the crane are established according to the motion equation of the Lagrange-Euler when the tower crane carry out the transition, rotation, lifting movement at the same time.

$(M+m) \ddot{\rho}-(M+m) \rho \dot{\psi}^{2}+m(l \ddot{\theta} \cos \varphi \cos \theta+$

$2 i \dot{\theta} \cos \varphi \cos \theta+\ddot{l} \cos \varphi \sin \theta-l \ddot{\varphi} \sin \varphi \sin \theta-$

$l \dot{\varphi}^{2} \cos \varphi \sin \theta-l \dot{\theta}^{2} \cos \varphi \sin \theta-2 \dot{l} \dot{\psi} \sin \varphi-$

$\left.l \ddot{\psi} \sin \varphi-l \dot{\psi} \dot{\varphi} \cos \varphi-\dot{\psi}^{2} l \cos \varphi \sin \theta\right)+b_{\rho} \dot{\rho}=F_{\rho}$.

$\left(J_{m}+M \rho^{2}+m \rho^{2}\right) \ddot{\psi}-2(M+m) \rho \dot{\rho} \dot{\psi}+m(\rho l \ddot{\varphi} \cos \varphi+$

$2 \rho \dot{l} \dot{\varphi} \cos \varphi+2 l \dot{l} \dot{\psi} \sin ^{2} \varphi+l^{2} \ddot{\psi} \sin ^{2} \varphi+$

$2 l^{2} \dot{\psi} \dot{\varphi} \sin \varphi \cos \varphi+2 l \dot{l} \dot{\varphi} \sin \theta+l^{2} \ddot{\varphi} \sin \theta+l^{2} \dot{\theta} \dot{\varphi} \cos \theta-$

$l \ddot{\rho} \sin \varphi+2 \rho l \ddot{\psi} \cos \varphi \sin \theta+l^{2} \ddot{\psi} \cos ^{2} \varphi \sin ^{2} \theta+$

$2 l \dot{\rho} \dot{\psi} \cos \varphi \sin \theta+2 \rho \dot{l} \dot{\psi} \cos \varphi \sin \theta+$

$2 \rho l \dot{\psi} \dot{\theta} \cos \varphi \cos \theta-2 \rho l \dot{\psi} \dot{\varphi} \sin \varphi \sin \theta+$

$2 l i \dot{\psi} \cos ^{2} \varphi \sin ^{2} \theta-\rho l \dot{\varphi}^{2} \sin \varphi-$

$2 l^{2} \dot{\psi} \dot{\varphi} \sin \varphi \cos \varphi \sin ^{2} \theta+2 l^{2} \dot{\psi} \dot{\theta} \cos ^{2} \varphi \sin \theta \cos \theta-$

$l^{2} \ddot{\theta} \sin \varphi \cos \varphi \cos \theta-2 l i \dot{\theta} \sin \varphi \cos \varphi \cos \theta+\rho \ddot{l} \sin \varphi+$

$l^{2} \dot{\theta}^{2} \sin \varphi \cos \varphi \sin \theta-l^{2} \dot{\theta} \dot{\varphi} \cos ^{2} \varphi \cos \theta+$

$\left.l^{2} \dot{\theta} \dot{\varphi} \sin ^{2} \varphi \cos \theta\right)+b_{\psi} \rho \dot{\psi}=F_{\psi}$.

$m(\ddot{l}-g \cos \varphi \cos \theta+\ddot{\rho} \cos \varphi \sin \theta+2 \dot{\rho} \dot{\psi} \sin \varphi+$

$\rho \ddot{\psi} \sin \varphi+\rho \dot{\psi} \dot{\theta} \cos \varphi-l \dot{\varphi}^{2}-l \dot{\theta}^{2} \cos ^{2} \varphi-l \dot{\psi}^{2} \sin ^{2} \varphi-$

$2 l \dot{\psi} \dot{\varphi} \sin \theta-\rho \dot{\psi}^{2} \cos \varphi \sin \theta-l \dot{\psi}^{2} \cos ^{2} \varphi \sin ^{2} \theta-$

$\rho \dot{\psi} \dot{\varphi} \cos \varphi+2 l \dot{\psi} \dot{\theta} \sin \varphi \cos \varphi \cos \theta)+b_{l} \dot{l}=F_{l}$.

$l \ddot{\theta} \cos \varphi+g \sin \theta+\ddot{\rho} \cos \theta-l \ddot{\psi} \sin \varphi \cos \theta-$

$\dot{\psi}^{2}(\rho+l \cos \varphi \sin \theta) \cos \theta+2 i \dot{\theta} \cos \varphi-2 l \dot{\varphi} \dot{\theta} \sin \varphi+$

$2 \dot{\rho} l \cos \theta-2 \dot{l} \dot{\psi} \sin \varphi \cos \theta-2 l \dot{\psi} \dot{\varphi} \cos \varphi \cos \theta=0$.

$l \ddot{\varphi}+g \sin \varphi \cos \theta-\ddot{\rho} \sin \varphi \sin \theta+\ddot{\psi}(l \sin \theta+\rho \cos \varphi)+$

$l \dot{\theta}^{2} \sin \varphi \cos \varphi+\dot{\psi}^{2} \sin \varphi\left(-l \cos \varphi \sin ^{2} \theta+\rho \sin \theta\right)+$

$2 \dot{l} \dot{\varphi}+2 \dot{l} \dot{\psi} \sin \theta+2 l \dot{\psi} \dot{\theta} \cos ^{2} \varphi \cos \theta+2 \dot{\rho} \dot{\psi} \cos \varphi=0$.

Where, Jm denotes moment of inertia of the hoisting jib. $\mathrm{M}$ and $\mathrm{m}$ respectively represent the trolley mass and cargo mass. b $\rho, b \psi$, and bl respectively denote the damping coefficients of transition motion, rotary motion, and hoisting motion. $\mathrm{F} \rho, \mathrm{F} \psi$, and $\mathrm{Fl}$ are respectively the driving forces of transition mechanism, rotation mechanism, and hoisting mechanism. The acceleration of gravity force is $\mathrm{g}$.

The dynamic models of the tower crane are composed of the dynamics equations of the transition mechanism, the rotating mechanism, the hoisting mechanism and the dynamics equations of the cargo oscillation. There are mutual coupling between the state variables. The crane system is a two order nonlinear time-varying differential system. The cargo swing equations (4) and (5) are two order oscillation sections about the swing angle $\theta$ and $\varphi$ that describe the relationship between the transition motion, rotary motion and cargo oscillation. The accelerations of the transition motion and rotary motion are the input of the section and the cargo swing angles are the output of the section. The hoisting and lowering motions make the section become a weakly damping system. The swing frequency is related to the length of the hoisting cable and the swing magnitude is related to the resultant acceleration.

Based on MATLAB simulation software, according to the nonlinear dynamics models of the crane $(1) \sim(5)$, the cargo oscillations are shown in Fig . 3 when the crane carry out transition, rotation, and hoisting motion at the same time.

Given the following conditions, the cargo mass $m=1 \mathrm{~kg}$, trolley mass is $M=1.5 \mathrm{~kg}$, the maximum acceleration of the trolley is amax $=1 \mathrm{~ms}-2$, the rated speed of the trolley $\mathrm{v}=0.3$ $\mathrm{ms}-1$, the equivalent damping coefficient $b \rho=0.1 \mathrm{~N} \mathrm{sm}-1$, the reference is speed up for $0.3 \mathrm{~s}$ before reaching the rated speed, run for $2 \mathrm{~s}$ at a constant speed, finally decelerate 0.3 $\mathrm{s}$ before stopping. The moment of inertia of hoisting jib is $\mathrm{Jm}=5.5 \mathrm{kgm} 2$, the rated rotary speed of rotation $\dot{\psi}_{\max }$ $=0.3$ rads -1 , the reference is speed up for $0.3 \mathrm{~s}$ before reaching the rated speed, run for $2 \mathrm{~s}$ at a constant speed, finally decelerate $0.3 \mathrm{~s}$ before stopping. Hoisting mechanism lifts from $0.8 \mathrm{~m}$ to $0.4 \mathrm{~m}$, and then lowers to $0.8 \mathrm{~m}$. That will realize point-to-point position control[7-8].

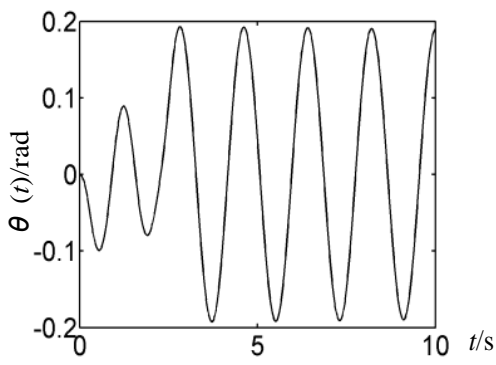

(a) Oscillation angle $\theta(t)$

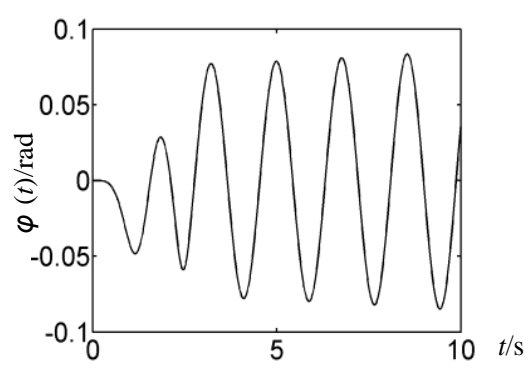

(b) Oscillation angle $\varphi(t)$

Figure 3. Cargo oscillation based on the nonlinear models.

According to the Z135 tower crane, we designed the simulation experiment system of tower crane in the ratio of $1: 30$ as in Fig .4. CCD camera is fixed on the trolley to record the position of the cargo relative to the cargo suspension point. The moving track of the cargo is calculated by the image processing algorithm and then the swing angle and swing angular velocity of the cargo were calculated. The angle difference of the measured value and 
the calculated values based on (4) and (5) are shown in Fig.5.

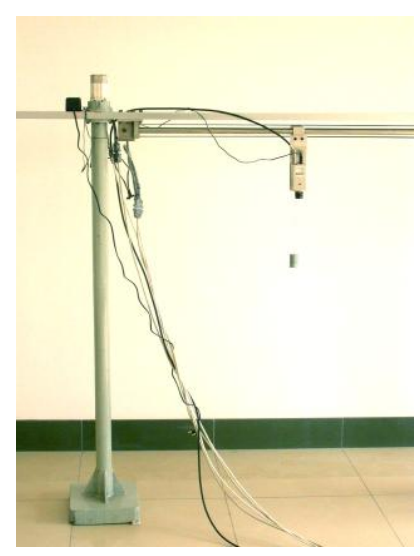

Experiment system of tower crane.

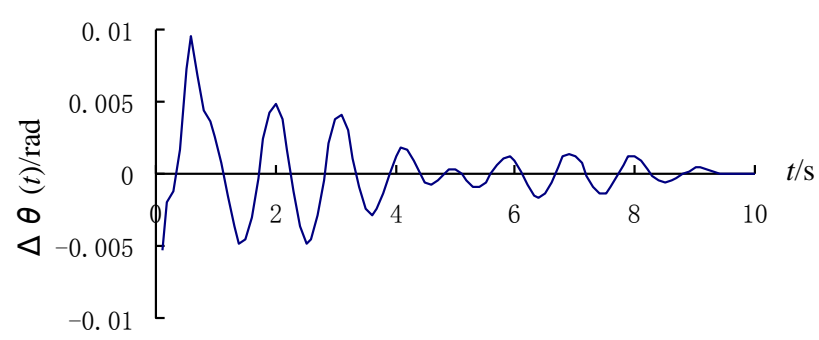

(a) The difference of the oscillation angle $\Delta \theta(t)$

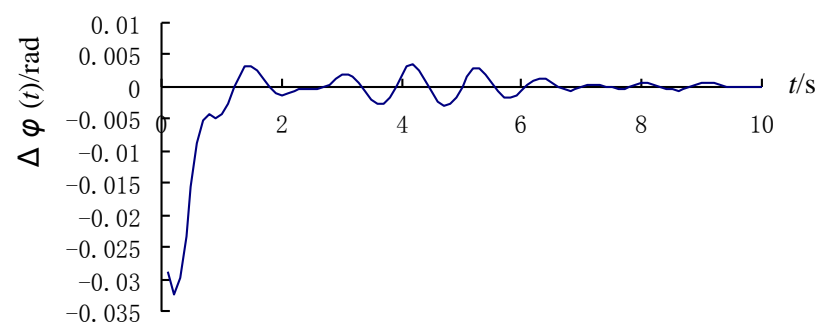

(b) The difference of the oscillation angle $\Delta \phi(t)$

Figure 4. The difference between the measured value of thecargo swing angle and the calculated value of the nonlinear models.

Because the models do not consider the delay characteristics of mechanical system and air resistance, the theoretical calculation value and the actual measurement value have some error. But the maximum error is not more than $30 \%$ and the error is becoming smaller and smaller, and finally converges to 0 . The experimental results show that the theoretical calculation value can gradually tracking the actual value and (4) and (5) can accurately describe the cargo swing characteristics.

\section{LINEAR MODELS OF TOWER CRANEDS}

Around the equilibrium state $\theta \mathrm{e}=0^{\circ}$ and $\varphi \mathrm{e}=0^{\circ}$, the linear models of the crane are following.

$$
M \ddot{\rho}-M \rho \dot{\psi}^{2}-m g \theta+b_{\rho} \rho=F_{\rho} .
$$

$\left(J_{m}+M \rho^{2}\right) \ddot{\psi}+2 M \rho \dot{\rho} \dot{\psi}-m \rho g \varphi+b_{\psi} \dot{\psi}=F_{\psi}$. $m(\ddot{l}-g)+b_{l} \dot{l}=F_{l}$.

$l \ddot{\theta}+2 \dot{l} \dot{\theta}+\left(g+l \dot{\psi}^{2}\right) \theta-2 l \dot{\psi} \dot{\varphi}-l \ddot{\psi} \varphi=-\ddot{\rho}+\rho \dot{\psi}^{2}-2 \dot{\rho} l$.

$l \ddot{\varphi}+2 \dot{l} \dot{\varphi}+\left(g-l \dot{\psi}^{2}\right) \varphi+2 l \dot{\psi} \dot{\theta}+l \ddot{\psi} \theta=-\rho \ddot{\psi}-2 \dot{\rho} \dot{\psi}$. iGiven the following conditions, the rated speed, run for $9.7 \mathrm{~s}$ at a constant speed, finally stop at the position $3.155 \mathrm{~m}$. The rated rotary angular speed of rotation $\omega=0.3 \mathrm{rad} / \mathrm{s}$, the reference is speed up for $0.3 \mathrm{~s}$ before reaching the rated speed, run for $9.7 \mathrm{~s}$ at a constant speed, finally stop at the position $2.955 \mathrm{rad}$. The hoisting mechanism lifts from 0.8 $\mathrm{m}$ to $1.5 \mathrm{~m}$, and then lowers to $0.8 \mathrm{~m}$. That will realize point-to-point position control. The cargo swing is shown in Fig .6.

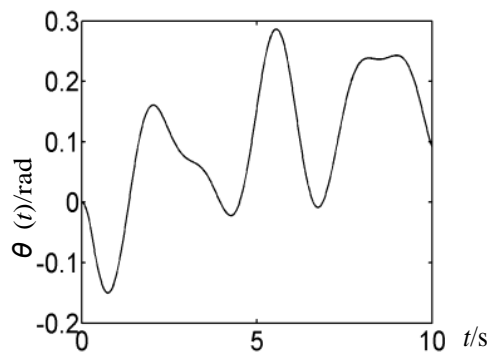

(a) Oscillation angle $\theta(t)$

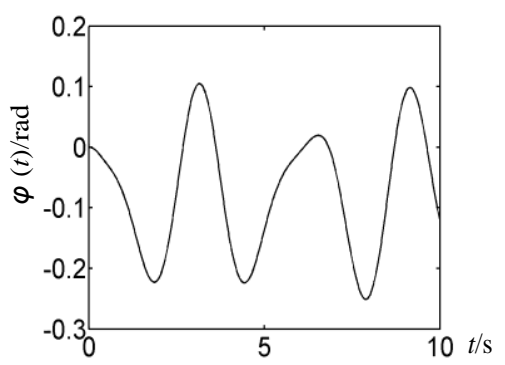

(b) Oscillation angle $\varphi(t)$

Figure 5. Cargo oscillation based on the linear models.

From Fig .6. it can be seen that the cargo swing center line is tilted along with increasing range of transition motion and rotation.

\section{ACTION OF CRANE DYNAMIC PARAMETERS ON THE CENTRAL LINE OF CARGO OSCILLATION}

The hoisting movement does not affect the position of the center line of the cargo oscillation, but has a decisive effect on the free oscillation time. The crane transition and rotation induce the center line of cargo oscillation to tilt and the tilt values are described with the equilibrium states $\theta \mathrm{e}$ and $\varphi \mathrm{e}$.

$$
\begin{gathered}
\theta_{\mathrm{e}}=\tan ^{-1}\left(\left(\rho \dot{\psi}^{2}-\ddot{\rho}\right) / \mathrm{g}\right){ }_{1 !} \\
\varphi_{\mathrm{e}}=\tan ^{-1}\left(-(\rho \ddot{\psi}+2 \dot{\rho} \dot{\psi}) / \sqrt{g^{2}+\left(\rho \dot{\psi}^{2}-\ddot{\rho}\right)^{2}}\right){ }_{1} . .
\end{gathered}
$$

Equation (11) and (12) describes the influence of dynamic parameters on the center line of the cargo oscillation. Namely the cargo oscillates in the inclined gravity field and the oscillation center line is the equilibrium states. 


\section{MAXIMUM AMPLITUDE OF CARGO OSCILLATION ANGLE CAUSED B Y INERTIA FORCES}

From the linear models we can see that the oscillation angle $\theta$ and $\varphi$ are mainly induced by the starting and braking inertia forces. In the shortest time of starting and braking, the largest input of linear models will produce the maximum oscillation angle and oscillation speed. Therefore we can estimate the maximum oscillation angle and oscillation speed. The linear model is simplified as the oscillation model acted by the inertial force of the transition and the inertia force of the rotary motion. for (8) is

$$
\begin{aligned}
& \ddot{\theta}+\mathrm{g} / l \cdot \theta=-\ddot{\rho} / l .{ }_{1} . \dot{\varphi} \\
& \ddot{\varphi}+\mathrm{g} / l \cdot \varphi=-\rho / l \cdot \ddot{\psi}{ }_{1} \text {.The general solution }
\end{aligned}
$$

$$
\theta(t)=c_{1} \cos \omega_{\mathrm{n}} t+c_{2} \sin \omega_{\mathrm{n}} t-\ddot{\rho} l \mathrm{~g} .
$$

Where, $\mathrm{c} 1$ and $\mathrm{c} 2$ are undetermined coefficients, $\omega n$ is the natural frequency of the cargo oscillation, $\omega_{\mathrm{n}}^{2}=\mathrm{g} / l$. Assuming that the initial state of the system is 0 , the solution of (15) is following.

$$
\theta(t)=\ddot{\rho} / \mathrm{g}\left(\cos \omega_{\mathrm{n}} t-1\right){ }_{1} \quad \dot{\theta}(t)=-\ddot{\rho} \omega_{\mathrm{n}} / \mathrm{g} \cdot \sin \omega_{\mathrm{n}} t ._{1}
$$

The sine and cosine functions are expanded based on Taylor series and the higher order terms are neglected. Starting in the shortest time, the cargo oscillation angle and velocity at the moment of finishing the starting are $\lim _{t \rightarrow 0} \theta(t)=0, \lim _{t \rightarrow 0} \dot{\theta}(t)=-v_{\max } / l$. In the same way, the maximum cargo oscillation speed of the rotary motion are $\dot{\theta}_{\max }=-v_{\max } / l, \dot{\varphi}_{\max }=-\rho \dot{\psi}_{\max } / l$. According to the law of conservation of energy, the maximum oscillation angle is estimated.

$$
\begin{aligned}
& \theta_{\max }=\dot{\theta}_{\max } / \omega_{\mathrm{n}}=-v_{\max } / \sqrt{\mathrm{g} l} . \\
& \varphi_{\max }=\dot{\varphi}_{\max } / \omega_{\mathrm{n}}=-\rho \dot{\psi}_{\max } / \sqrt{\mathrm{g} l} .
\end{aligned}
$$

Through the above derivation, if the hoisting cable length and transition speed meet the condition $l<\left(18 v_{\max }\right)^{2} /\left(\pi^{2} g\right)$, the oscillation angle $\theta$ is greater than $10^{\circ}$, if the hoisting cable length and rotary speed meet $l<\left(18 \rho \dot{\psi}_{\max }\right)^{2} /\left(\pi^{2} \mathrm{~g}\right)$ than $10^{\circ}$. This does not meet model and the linear model has large error. Equation (18) and (19) reveal the influence rule of the dynamic parameters on the maximum oscillation angle of the cargo.

Through the above derivation, if the hoisting cable length and transition speed meet the condition $l<\left(18 v_{\max }\right)^{2} /\left(\pi^{2} \mathrm{~g}\right)$, the oscillation angle $\theta$ is greater than $10^{\circ}$, if the hoisting cable length and rotary speed meet $l<\left(18 \rho \dot{\psi}_{\max }\right)^{2} /\left(\pi^{2} g\right)$, the oscillation angle $\varphi$ is greater than $10^{\circ}$. This does not meet the assumptions of the linear model and the linear model has large error. Equation (18) and (19) reveal the influence rule of the dynamic parameters on the maximum oscillation angle of the cargo.

\section{CONCLUSIONS}

With the tower crane transition motion and rotary motion, centrifugal force and Coriolis force increase and oscillation angles gradually deviate from the equilibrium position, the oscillation center line tilt, no longer along the vertical direction, the linear model has greater error. When the dynamic parameters exceed a certain value, the center line deviates from the equilibrium position and no longer meets the linear condition. It is necessary to recalculate the equilibrium states, modify the linear models as the controlled object model.

\section{ACKNOWLEDGMENT}

This work was financially supported by the National Natural Science Foundation of China (Grant No. 51475277).

\section{REFERENCES}

[1] Jie Huang, Xumiao Xie, and Zan Liang. Control of Bridge Cranes With Distributed-Mass Payload Dynamics. IEEE/ASME Transactions on Mechatronics Vol. 20, No.1, February, 2015:481486.

[2] Andreas Rauh, Luise Senkel, Jovanka Gebhardt, Harald Aschemann. Stochastic Methods for the Control of Crane Systems in Marine Applications. 2014 European Control Conference (ECC), June 24-27, 2014, Strasbourg, France,2014:2998-3003.

[3] Jisup Yoon, Shelley Nation, William Singhose, and Joshua Eric Vaughan. Control of Crane Payloads That Bounce During Hoisting. IEEE Transactions on Control Systems Technology, Vol. 22, No. 3, May, 2014:1233-1238.

[4] Fabrizio Padula, Antonio Visioli, Domenico Facchinetti, and Alberto Saleri. A Dynamic Inversion Approach for Oscillation-free Control of Overhead Cranes. Emerging Technologies \& Factory Automation (ETFA), 2015 IEEE 20th Conference, Luxembourg. Sept. 20158-11 Sept. 2015:1-6

[5] Mingxiao Dong. Time-delayed filter theory and engineering application. Beijing, Science Press. 2008,3.

[6] Yongchun Fang, Pengcheng Wang, Ning Sun, and Yichun Zhang. Dynamics Analysis and Nonlinear Control of an Offshore Boom Crane. IEEE Transactions on Industrial Electronics, Vol. 61, No. 1, January, 2014:414-427.

[7] Dongho Kim, Youngjin Park. Tracking control in x-y plane of an offshore container crane. Journal of Vibration and Control. 2015, July 1:1-15.

[8] Daichi Fujioka, Manan Shah, William Singhose. Robustness Analysis of Input-Shaped Model Reference Control on aDoublePendulum Crane.2015 American Control Conference Palmer House Hilton, Chicago, IL, USA. July 1-3, 2015:2561-2566. 